\title{
Relationship between Physical Disability and Black Holes in Multiple Sclerosis: Upper Extremity Functions - an Important Parameter
}

\author{
(D) Bilge Piri Cinar ${ }^{1}$, (D) Gulcan Kalaycı ${ }^{1}$, (D) Mustafa Acıkgoz¹ , (D) Serkan Ozakbas², \\ 'Zonguldak Bulent Ecevit University Faculty of Medicine, Department of Neurology, Zonguldak, Turkey \\ 2Dokuz Eylul University Faculty of Medicine, Department of Neurology, Izmir, Turkey
}

\section{Abstract}

Objective: Typical lesion locations in multiple sclerosis (MS) and hypointense lesions that persist > 6 months on T1-weighted magnetic resonance imaging (MRI) are known as black holes. This study aimed to investigate the potential relationship between brain MRI lesion localization and the presence of black holes and upper extremity function in patients with MS in remission.

Materials and Methods: The nine-hole peg test (NHPT), timed 25-foot walk test (T25FWT), and expanded disability status scale (EDSS) scores were calculated with neurological examinations in the period when MRI was performed ( \pm 1 week).

Results: This study included 47 patients diagnosed with MS. The mean patient age was 34.24 \pm 8.38 (19-54) years. The mean EDSS score of the study group was $1.7 \pm 0.85$ (0-3). The mean NHPT time was $17.27 \pm 2.70 \mathrm{~s}$ (13.74-27.23 s). The mean T25FWT time of the study group was $4.35 \pm 0.62 \mathrm{~s}$ (3.306.05 s). Juxtacortical lesions were present in 46 (97.0\%) patients, cortical lesions in 2 (4.3\%), brain stem lesions in 38 (80.9\%), and cerebellar lesions in 16 (34\%). Permanent black holes were found in 37 (78.7\%) patients, with more than one black holes being detected in 33 of these patients. The mean NHPT time was significantly longer in patients with permanent black holes detected by MRI of the brain than in those without black holes $(p=0.034)$. A weak correlation was found between the EDSS score and NHPT time $(r=0.192, p=0.046)$.

Conclusion: The presence and number of permanent black holes can be used as a marker of disability. Upper extremity functions may well represent disability, especially in patients with low EDSS scores.

Keywords: Multiple sclerosis, physical disability, upper extremity functions, black holes

\section{Introduction}

Various clinical symptoms may be seen in multiple sclerosis (MS), depending on the affected region of the central nervous system, such as weakness in one or more extremities, optic neuritis, paresthesia, diplopia, and bladder problems (1). The first clinical manifestation that produces findings such as medulla spinalis involvement, isolated optic neuropathy, or hemisphere involvement in which MS-specific lesions are observed by magnetic resonance imaging (MRI), but the spread criteria are not met at that time, is known as a clinically isolated syndrome (CIS) $(1,2)$. MRI is crucial in the timing of diagnosis of conversion from CIS to MS and in monitoring the disease course and effectiveness of treatment (3). While lesions can be seen in different locations, periventricular, corpus callosum, juxtacortical, brainstem, spinal cord, temporal lobe, optic nerve, cerebellum, and cerebellar peduncle involvement is typical for MS (4). Although the morphological characteristics of the lesions have been described in greater detail in recent years (5), typical features are an ovoid shape, perpendicularity to the ventricle, and size $>3 \mathrm{~mm}$ (4).

In addition to the typical lesion locations in MS, hypointense lesions persisting for $>6$ months on T1-weighted MRI are known as "black holes," and these show permanent axon loss (6). The Barkhof-Tintore criteria employed to evaluate MRI lesions in the diagnosis of MS were subsequently revised, and the Swanton criteria began its implementation through a modification of the 2010 McDonald criteria. However, black holes were not included in either the McDonald or MAGNIMS MRI diagnostic

Address for Correspondence: Bilge Piri Cinar, Zonguldak Bulent Ecevit University Faculty of Medicine, Department of Neurology, Zonguldak, Turkey E-mail: bilge.cinarpiri@gmail.com.tr ORCID-ID: orcid.org/0000-0002-4884-0717

Received: 14.06.2021 Accepted: 01.08.2021

${ }^{\circ}$ Copyright 2021 by the Journal of Multiple Sclerosis Research published by Galenos Publishing House. 
criteria. However, the presence, numbers, and volume of black holes are thought to be important in patient follow-up because of their relatively simple evaluation and close association with a physical disability. Although upper and lower extremity functions are closely related to physical disability in MS, lower extremity functions are more markedly affected at higher expanded disability status scale (EDSS) scores, while upper extremity functions are often overlooked. In a previous study, EDSS and upper extremity functions were found to be more significantly associated with lower extremity functions (7). The present study investigated the potential relationship between brain MRI lesion localization and the presence of black holes and both upper and lower extremity functions in patients with MS in remission.

\section{Materials and Methods}

\section{Patient Enrolment}

Patients under follow-up in our MS clinic and diagnosed with relapsing-remitting MS (RRMS) according to the McDonald 2017 criteria but in the remission period were included in the study. The remission period is defined as MS that is not in the attack or progression period, at least 3 months after the attack. In this cross-sectional study, patients who underwent brain MRI performed with a 1.5 Tesla scanner were routinely evaluated in the remission period using T2-weighted axial, sagittal, fluidattenuated inversion recovery (FLAIR) axial, and T1 axial sections. Lesion localizations at MRI were classified as periventricular, juxtacortical, cortical, brainstem, and cerebellar lesions, and the presence of black holes was also assessed. Spinal lesions were not included in the study, since not all patients had cervical MRI data, and the relationship between black hole presence and disability was investigated.

All procedures performed in studies involving human participants were in accordance with the ethical standards of the institutional and/or national research committee and with the 1964 Helsinki Declaration and its later amendments or comparable ethical standards. The data was obtained from our hospital records prospectively for the study. Ethics approval was obtained from the ethics committee of Dokuz Eylül University and written informed consent was obtained from the patients.

Various scales were employed to evaluate disability during patient follow-up, and the most widely used was the EDSS (8). EDSS scores were calculated as a value between 0 and 10 based on functional scoring, including visual, brainstem, cerebellar, pyramidal, bowel, and bladder functions, sensory functions, and ambulation (9). One of the principal disadvantages of the EDSS, used in MS for many years, is that upper and lower extremity functions cannot be separately evaluated.

The nine-hole peg test (NHPT) is regarded as the gold standard in the assessment of upper extremity skills and is frequently applied to patients with MS (10). The timed 25 -foot walk test (T25FWT) is employed to evaluate the lower extremity skills of patients with MS (11). In this study, the EDSS scores of the patients were calculated with neurological examinations in the period when MRI was performed ( \pm 1 week). Moreover, NHPT was assessed for upper extremities, and the mean times were noted. The T25FWT was repeated twice, and the mean times were recorded. All patients were examined and physically evaluated by a single physician, and MR images were evaluated blindly by another physician (both physicians are MS specialists).

\section{Statistics Analyses}

Obtained data were statistically analyzed using the SPSS 19.0 package program. Descriptive statistics of the study variables are shown as mean, standard deviation, and minimum-maximum values, and descriptive statistics of categorical variables are shown as frequency and percentage. The conformity of the variables to the normal distribution was examined by both visual and normal distribution tests. Independent sample t-test analysis was used for two-group comparisons of normally distributed variables, and the chi-square test was used for categorical variables. The relationship between continuous variables was analyzed by Pearson or Spearman correlation analysis when necessary, according to its suitability for normal distribution; $p$-value $<0.05$ indicated significance.

\section{Results}

The study included 47 patients diagnosed with MS based on the McDonald 2017 criteria and currently in remission (F/M, 38/9). The mean patient age was $34.24 \pm 8.38$ (19-54) years. The first clinical event was polysymptomatic in $59.6 \%$ of the patients. Disease onset with brainstem, motor, cerebellar, and sphincter involvement, a poor prognostic factor marker, was noted in 31 (66\%) cases. The mean disease duration of the study group was $19.08 \pm 12.22$ months, and the mean EDSS score was 1.7 \pm 0.85 (03) points. Upper and lower extremity functions were evaluated using the NHPT and T25FWT, respectively. The mean NHPT time of the patients was 17.27 \pm 2.70 (13.74-27.23). The mean T25FWT time of the study group was $4.35 \pm 0.62 \mathrm{~s}(3.30-6.05 \mathrm{~s})$.

Brain MRI examinations were performed using T2-weighted axial, sagittal, FLAIR axial, and T1 axial sections. Periventricular lesions were present in all patients. Juxtacortical lesions were found in 46 (97.0\%) patients, cortical lesions in 2 (4.3\%), brain stem lesions in 38 (80.9\%), and cerebellar lesions in 16 (34\%). Permanent black holes were found in 37 (78.7\%) patients, with more than one black holes being detected in 33 of these (89.1\%) (Figure 1). When the group with black holes and the group without black holes were compared in terms of disease duration, no significant difference was observed between the two groups $(p=0.08)$. 
Journal of Multiple Sclerosis Research 2021;1(1):17-21

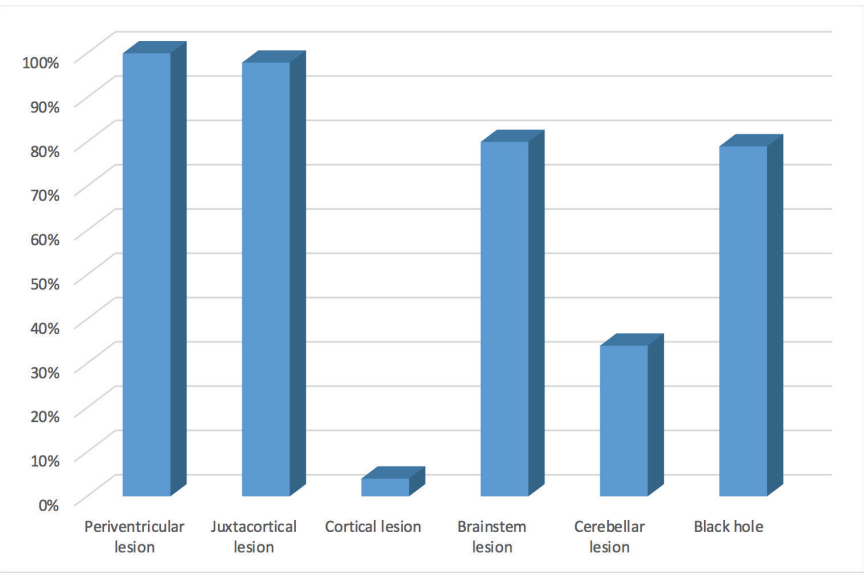

Figure 1. Brain MRI lesion characteristics of the study group

MRI: Magnetic resonance imaging

The mean NHPT time was significantly longer in patients with permanent black holes detected by brain MRI than in those without black holes $(p=0.034)$. A weak correlation was determined between the EDSS score and NHPT $(r=0.192$, $p=0.046$ ). No significant difference was found in the T25FWT times between patients with and without permanent black holes ( $p=0.062$ ). No significant correlation was observed between EDSS and T25FWT time $(p=0.058)$. In addition, no significant difference was found in lesion localization and physical parameters in MRI.

\section{Discussion}

MRI is crucial in the diagnosis and follow-up of MS and is one of the main guides in both prognosis and evaluation of treatment response. Although T2 hyperintense lesions detected at MRI are critical in the diagnosis of MS, their correlation with both physical and cognitive disability is low (12-14). Moreover, although the number of 2 hyperintense lesions at disease onset gives some indications about the prognosis, the association of lesion burden with clinical manifestations decreases as the disease progresses (12). T1 hypointense lesions, a powerful indicator of axonal damage and demyelination, that exist $>6$ months are known as black holes, and studies have revealed a closer relationship between physical disability and T2 hyperintense lesions (15). A relationship has been shown between the intensity of the hypointensity and axonal damage, and a study concluded that axonal damage increases in line with hypointensity (16). In addition, a potential association was found between the intensity of T1 hypointense lesions and EDSS (16). Previous studies have shown that an increase in the number or volume of black holes is associated with a poor clinical course, the T1 lesion burden is associated with EDSS scores, and increasing T1 lesions are associated with a worsening clinical course (17).

Although physical disability in MS is most frequently evaluated using the EDSS, this system has several limitations, such as the
Piri Cinar et al. Relationship Between Physical Disability and Black Holes in MS

fact that it does not assess the cognitive field or upper and lower extremity functions. However, the evaluation and regular monitoring of both upper and lower extremity functions are significant in clinical follow-up. In addition, lower upper extremity functions can be used in combination with the EDSS when evaluating physical disability in MS. In a previous study, patients with MS (pwMS) with two fundamental motor tasks were evaluated and compared with healthy controls; pwMS performed worse than HC on both tasks. Moreover, upper and lower extremity motor skills in pwMS worsen as disability increases (18). In another study, progressive decline in lower extremity motor performance and hand functions with each increase in EDSS score suggests that 9-HPT is a good marker of decreased hand function and should be included in the clinical monitoring of patients (19).

In the present study, upper and lower extremity functions were evaluated with 9-HPT and T25FWT. These parameters and the relationship between EDSS and presence of black holes were examined. In this study, while no connection was detected between black holes, regarded as evidence of neurodegeneration, and EDSS, a significant correlation was found between the presence of permanent black holes and NHPT used to assess upper extremity functions. The absence of any association between EDSS and permanent black holes may be due to the inadequacy of EDSS in evaluating upper extremity functions. Alternatively, this may be due to our patient's low physical disability scores assessed using the EDSS. In the present study, no relationship was determined between black holes and lower extremity functions, an important physical status parameter. While this can be explained by the low physical disability scores of the study group, it can also be regarded as a finding: although T25FWT is used for lower extremity functions, it is insufficient for showing lower limb capacity despite indicating lower extremity performance. A study evaluating the association of several imaging modalities with worsening clinical disability scores over 10 years in RRMS showed that the number and volume of permanent black holes exhibited the best performance (20).

In addition, imaging and neuropathology comparisons of more than 100 MS lesions revealed a powerful association of the degree of hypointensity with axonal density (15).

Contrast-enhancing lesions detected by MRI are also significant in the diagnosis and follow-up of MS since they are employed as evidence of spread over time in MS diagnostic criteria and as an indicator of disease activation. However, when performing contrast-enhanced MRI, it is difficult to evaluate betweenwhiles because of challenges in the application, such as the dose and timing of administration of contrast agent. No biomarker of neurodegeneration, which plays an important role in the progression and for which treatments are often inadequate, has emerged other than contrast-enhancing lesions, regarded 
as evidence of disease activation and inflammation in MS. The presence of permanent black holes has the potential for use as a marker of neurodegeneration and as an outcome measure in trials of potential protective agents. In a previous study, black hole development was evaluated over the course of 3-year interferon beta-1 $\mathrm{b}$ therapy in an RRMS group, and the number of contrast-enhancing lesions after 1 year of treatment was found to predict the change in black hole volume over the following 2 years. In that study, the black hole volume remained stable in patients without contrast-enhancing lesions but increased in the patient group with contrast-enhancing lesions (21). In the present study, contrast-enhancing lesion analysis could not be performed because not all patients had contrast-enhanced imaging, and this was considered as a limitation of this study.

Greater use of the detection of permanent black holes and even their degree of intensity are expected, which can be performed using standard T1-weighted images and commercial imaging software when performing MRI in the follow-up of the disease course or treatment of MS. An assembly of MS specialists in 2008 concluded that black holes, optical coherence tomography, brain volume, and magnetization transfer rate are criteria that can show neurodegeneration (22). These criteria may be employed more frequently for follow-up purposes and as a progression indicator in the future clinical follow-up of patients with MS. However, a standardized approach can be established by demonstrating the correlation of black holes, indicators of permanent axonal loss, with both physical and cognitive parameters by studies involving large sample sizes.

\section{Study Limitation}

Beside of the small number of patients in this study is that spinal lesions cannot be included when assessing upper extremity functions because not all patients have undergone a spinal MRI.

\section{Conclusion}

Upper extremity functions are often overlooked during routine evaluation. This may be related to the need for equipment for its assessment and the insufficient attention to upper extremity functions. However, the contribution of upper extremity functions, which significantly affect the daily life of an individual, to the disability of the disease should not be ignored. In addition, showing the relationship between upper extremity functions and the presence of black holes, which is a poor prognostic marker, is important, especially in pwMS with low EDSS, and our findings can draw attention to upper extremity functions.

\section{Ethics}

Ethics Committee Approval: Ethics approval was obtained from the ethics committee of Dokuz Eylül University.

Informed Consent: Informed consent from all participiants was obtained for the study.

\section{Authorship Contributions}

Surgical and Medical Practices: B.P.C., G.K., Concept: B.P.C., M.A., S.O., Design: B.P.C., M.A., S.O., Data Collection or Processing: B.P.C., G.K., S.O., Analysis or Interpretation: B.P.C., S.O., Literature Search: B.P.C., G.K., M.A., S.O., Writing: B.P.C., G.K., M.A., S.O.

Conflict of Interest: No conflict of interest was declared by the authors.

Financial Disclosure: The authors declared that this study received no financial support.

\section{References}

1. Garg N, Smith TW. An update on immunopathogenesis, diagnosis, and treatment of multiple sclerosis. Brain Behav 2015;5:e00362. doi: 10.1002/ brb3.362.

2. Klineova S, Lublin FD. Clinical course of multiple Sclerosis. Cold Spring Harb Perspect Med 2018;8:a028928. doi: 10.1101/cshperspect.a028928.

3. Wattjes MP, Rovira À, Miller D, Yousry TA, Sormani MP, de Stefano MP, Tintoré M, Auger C, Tur C, Filippi M, Rocca MA, Fazekas F, Kappos L, Polman C, Barkhof F, Montalban X; MAGNIMS study group. MAGNIMS consensus guidelines on the use of MRI in multiple sclerosis-establishing disease prognosis and monitoring patients. Nat Rev Neurol 2015;11:597-606

4. Giorgio A, De Stefano N. Effective utilization of MRI in the diagnosis and management of multiple sclerosis. Neurol Clin 2018;36:27-34.

5. Sivakolundu DK, Hansen MR, West KL, Wang Y, Stanley T, Wilson A, McCreary M, Turner MP, Pinho MC, Newton BD, Guo X, Rypma B, Okuda DT. Threedimensional lesion phenotyping and physiologic characterization Inform remyelination ability in multiple sclerosis. J Neuroimaging 2019;29:605-614.

6. Adusumilli G, Trinkaus K, Sun P, Lancia S, Viox JD, Wen J, Naismith RT, Cross $\mathrm{AH}$. Intensity ratio to improve black hole assessment in multiple sclerosis. Mult Scler Relat Disord 2018;19:140-147.

7. Alonso R, Eizaguirre MB, Silva B, Pita MC, Cohen L, Vanotti S, Garcea D. Upper limbs impairment in patients with multiple sclerosis: a relevant and currently undervalued measure. (P3.2-026). Neurology Apr 2019;92(Suppl 15):P3.2-026.

8. Solaro C, Grange E, Di Giovanni R, Cattaneo D, Bertoni R, Prosperini L, Messmer Uccelli M, Marengo D. Nine Hole Peg Test asymmetry in refining upper limb assessment in multiple sclerosis. Mult Scler Relat Disord 2020:45:102422. doi: 10.1016/j.msard.2020.102422.

9. Şen S. Neurostatus and EDSS calculation with Cases. Noro Psikiyatr Ars 2018;55(Suppl 1):S80-S83.

10. Feys P, Lamers I, Francis G, Benedict R, Phillips G, LaRocca N, Hudson LD, Rudick R. Multiple Sclerosis Outcome Assessments Consortium.: The NineHole Peg Test as a manual dexterity performance measure for multiple sclerosis. Mult Scler. 2017;23:711-720.

11. Sikes EM, Cederberg KL, Sandroff BM, Bartolucci A, Motl RW. Quantitative synthesis of timed 25-foot walk performance in multiple sclerosis. Arch Phys Med Rehabil 2020;101:524-534.

12. Göçmen R. The relevance of neuroimaging findings to physical disability in multiple Sclerosis. Noro Psikiyatr Ars 2018;55(Suppl 1): S31-S36.

13. Bastianello S, Giugni E, Amato MP, Tola MR, Trojano M, Galletti S, Luccichenti G, Quarantelli M, Picconi O, Patti F; COGIMUS study group. Changes in magnetic resonance imaging disease measures over 3 years in mildly disabled patients with relapsing-remitting multiple sclerosis receiving interferon $\beta-1 a$ in the COGnitive Impairment in MUltiple Sclerosis (COGIMUS) study. BMC Neurol 2011;11:125.

14. Bagnato F, Salman Z, Kane R, Auh S, Cantor FK, Ehrmantraut M, Gallo A Ikonomidou VN, Ohayon J, Pellicano C, Stern SK, McFarland HF. T1 cortical hypointensities and their association with cognitive disability in multiple sclerosis. Mult Scler 2010;16:1203-1212. 
15. van Waesberghe $J H$, Kamphorst W, De Groot CJ, van Walderveen MA Castelijns JA, Ravid R, Lycklama à Nijeholt GJ, van der Valk P, Polman $\mathrm{CH}_{\text {, }}$ Thompson AJ, Barkhof F. Axonal loss in multiple sclerosis lesions: magnetic resonance imaging insights into substrates of disability. Ann Neurol 1999;46:747-754.

16. Tam RC, Traboulsee A, Riddehough A, Sheikhzadeh F, Li DK. The impact of intensity variations in T1-hypointense lesions on clinical correlations in multiple sclerosis. Mult Scler 2011;17:949-957.

17. Truyen $L$, van Waesberghe $J H$, van Walderveen MA, van Oosten BW, Polman $\mathrm{CH}$, Hommes OR, Adèr HJ, Barkhof F. Accumulation of hypointense lesions ("black holes") on T1 spin-echo MRI correlates with disease progression in multiple sclerosis. Neurology. 1996;47:1469-1476.

18. Coghe G, Corona F, Pilloni G, Porta M, Frau J, Lorefice L, Fenu G, Cocco E, Pau $M$. Is there any relationship between upper and lower limb Impairments in people with multiple sclerosis? a kinematic quantitative analysis. Mult Scler Int 2019 Oct 9;2019:9149201. doi: 10.1155/2019/9149201.
19. Rammohan K, Li D, Halper J, McCurdy Murphy S, Wallace T, Patton L, Chrisant S, Zheng C, Khurana S. (EPI02) Motor Impairment in Multiple Sclerosis: Analysis from the North American Registry for Care and Research in Multiple Sclerosis (NARCRMS). International Journal of MS Care. 2020 May 2;22.

20. Giorgio A, Stromillo ML, Bartolozzi ML, Rossi F, Battaglini M, De Leucio A, Guidi L, Maritato P, Portaccio E, Sormani MP, Amato MP, De Stefano N. Relevance of hypointense brain MRI lesions for long-term worsening of clinical disability in relapsing multiple sclerosis. Mult Scler 2014;20:214-219.

21. Morgen K, Crawford AL, Stone RD, Martin R, Richert ND, Frank JA, McFarland HF. Contrast-enhanced MRI lesions during treatment with interferonbeta$1 \mathrm{~b}$ predict increase in $\mathrm{T} 1$ black hole volume in patients with relapsingremitting multiple sclerosis. Mult Scler 2005;11:146-148.

22. Adusumilli G, Trinkaus K, Sun P, Lancia S, Viox JD, Wen J, Naismith RT, Cross AH.: Intensity ratio to improve black hole assessment in multiple sclerosis. Mult Scler Relat Disord 2018;19:140. 